\title{
Low temperature spin-glass magnetic behavior of $\mathrm{Ce}_{3} \mathrm{Pd}_{20} \mathrm{Ge}_{6}$
}

\author{
Yu. P. Gaidukova, V. N. Nikiforova, and Yu. A. Koksharova \\ Low Temp. Dep., Faculty of Physics, M. V. Lomonosov Moscow State University, Russia \\ R. Szymczakb and H. Szymczakb
}

Institute of Physics, Polish Academy of Sciences, 02-668 Warsaw, Poland

A. V. Gribanov

Faculty of Chemistry, M. V. Lomonosov Moscow State University, Russia

Received October 14, 1997, revised March 3, 1998

\begin{abstract}
A polycrystalline sample of $\mathrm{Ce}_{3} \mathrm{Pd}_{20} \mathrm{Ge}_{6}$ is investigated using a dc SQUID magnetometer. A noticeable difference between $\mathrm{ZFC}$ and $\mathrm{FC}$ magnetic susceptibility is found below $\approx 60 \mathrm{~K}$. The temperature dependence of the magnetic susceptibility demonstrates an anomaly near approximately $2.8 \mathrm{~K}$ below which a remanent magnetic moment exists. Two characteristic temperatures detected support the assumption that there are different cerium magnetic subsystems in $\mathrm{Ce}_{3} \mathrm{Pd}_{20} \mathrm{X}_{6}(\mathrm{X}=\mathrm{Ge}, \mathrm{Si})$ compounds. Unusual magnetic behavior observed in $\mathrm{Ce}_{3} \mathrm{Pd}_{20} \mathrm{Ge}_{6}$ is discussed within the framework of the molecular magnetism' model which predicts a frustration of exchange interactions.
\end{abstract}

PACS:

\section{Introduction}

The problem of the coexistence of magnetic and Kondo ground states in $f$-electron systems has been discussed intensively over the past decade Ref. 1. In our previous works $[2,3]$ we reported on a Kondolike dependence of electrical resistivity and unusual magnetic behavior in polycrystalline samples of $\mathrm{Ce}_{3} \mathrm{Pd}_{20} \mathrm{X}_{6}(\mathrm{X}=\mathrm{Ge}, \mathrm{Si})$. The electrical resistivity of these compounds demonstrates logarithmic increasing below characteristic temperatures of $10 \mathrm{~K}$ $\left(\mathrm{Ce}_{3} \mathrm{Pd}_{20} \mathrm{Ge}_{6}\right)$ and $50 \mathrm{~K}\left(\mathrm{Ce}_{3} \mathrm{Pd}_{20} \mathrm{Si}_{6}\right)$. In autism a magnetic anomaly was found in $\mathrm{Ce}_{3} \mathrm{Pd}_{20} \mathrm{Si}_{6}$ below $\approx 50-60 \mathrm{~K}$. Though the crystal structures of $\mathrm{Ce}_{3} \mathrm{Pd}_{20} \mathrm{Ge}_{6}$ and $\mathrm{Ce}_{3} \mathrm{Pd}_{20} \mathrm{Si}_{6}$ are very similar, no magnetic anomalies were detected in the former compound above the helium temperature $([2,3])$. However, an antiferromagnetic-like peculiarity of ac-susceptibility in $\mathrm{Ce}_{3} \mathrm{Pd}_{20} \mathrm{Ge}_{6}$ was found at $1 \mathrm{~K}$ ([4]). In the presented work a polycrystalline sample of $\mathrm{Ce}_{3} \mathrm{Pd}_{20} \mathrm{Ge}_{6}$ has been investigated using a $\mathrm{dc}$ SQUID magnetometer in the temperature range from 2 to $200 \mathrm{~K}$ and at magnetic fields (250) KOe. The sample of $\mathrm{Ce}_{3} \mathrm{Pd}_{20} \mathrm{Ge}_{6}$ was prepared using a melting technique in arc furnace in an argon atmosphere as described in [5]. Annealing in the argon atmosphere (at pressure of $1.1 \cdot 10^{5} \mathrm{~Pa}$ ) was performed at $600{ }^{\circ} \mathrm{C}$ during about $700 \mathrm{~h}$. The crystal structure of the sample determined by the $\mathrm{x}$-ray analysis is the same as reported in [5]. The temperature dependence of the electrical resistivity of the sample being studied as a test shows a logarithmic increase below $10 \mathrm{~K}$ that accords with results obtained in $[2,3]$.

\section{Experimental results}

Figure 1 represents the temperature dependences of a static magnetic susceptibility at low (12 Oe) and high $(50 \mathrm{KOe})$ magnetic fields $\left(\chi_{\text {low }}\right.$ and $\chi_{\text {high }}$ , respectively). At high temperatures the following relationship takes place: $\chi_{\text {low }}>\chi_{\text {high }}$. Below approximately $10 \mathrm{~K} \chi_{\text {high }}$ is almost equal to $\chi_{\text {low }}$. It is interesting to note that the electrical resistivity has a Kondo minimum at the same temperature [2,3]. The solid line in Fig. 1 shows the Curie law's magnetic susceptibility $\chi_{\text {free }}$ of $\mathrm{Ce}^{3+}$ ions in $\mathrm{Ce}_{3} \mathrm{Pd}_{20} \mathrm{Ge}_{6}$ if they would be free of exchange interactions. The curves $\chi_{\text {low }}(T)$ and $\chi_{\text {free }}(T)$ cross each other at the temperature $\approx 50 \mathrm{~K}$. A is likely 


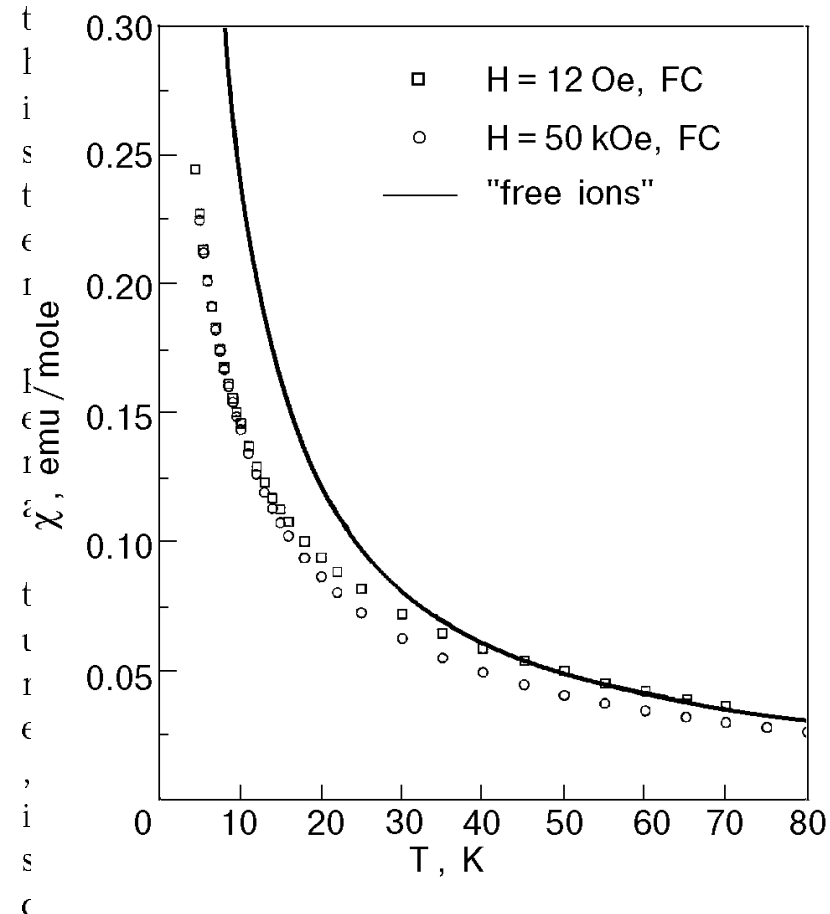

Fig. 1. Temperature dependences of $\mathrm{Ce}_{3} \mathrm{Pd}_{20} \mathrm{Ge}_{6}$ magnetic susceptibility at different magnetic fields. Solid line represents calculated «free ions» magnetic susceptibility which follows the Curie law.

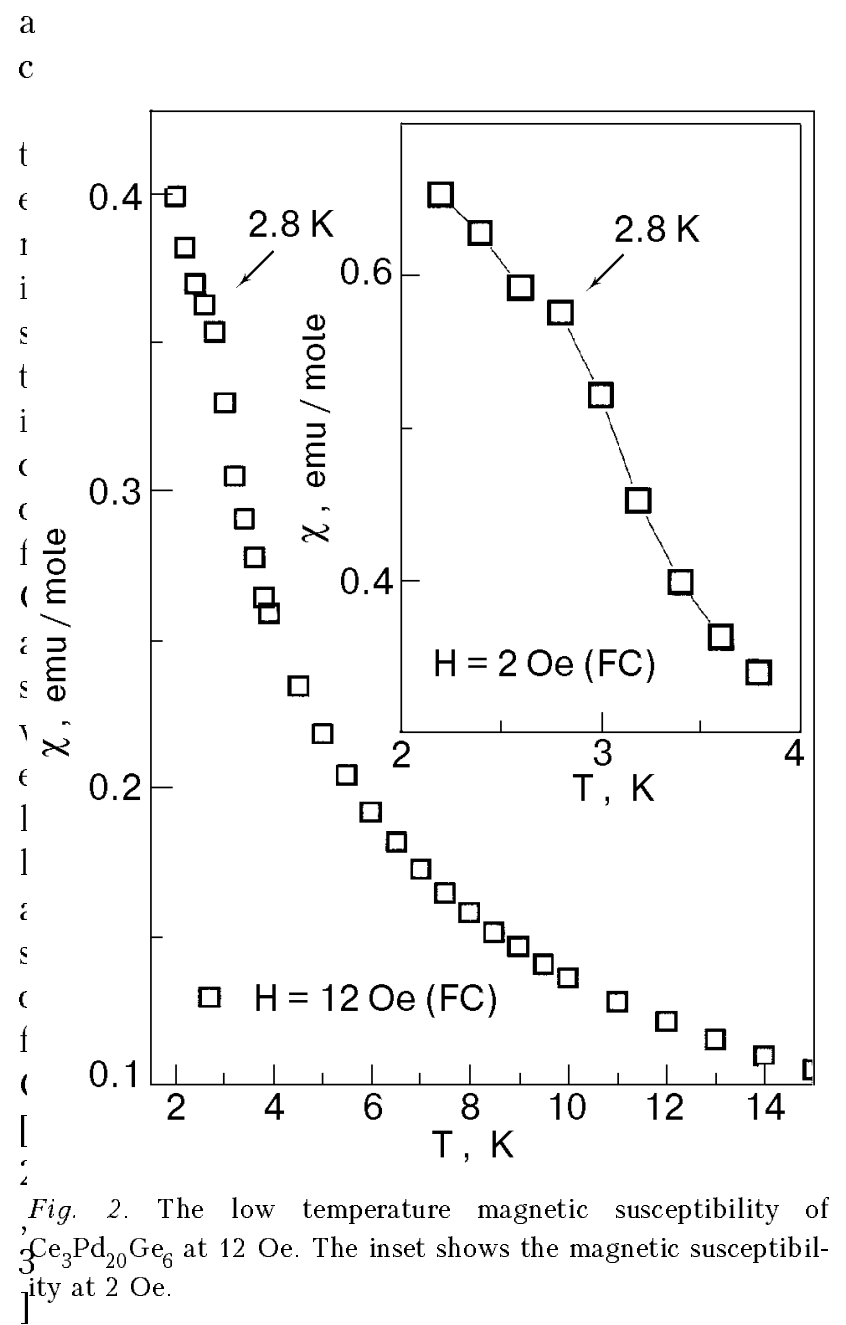

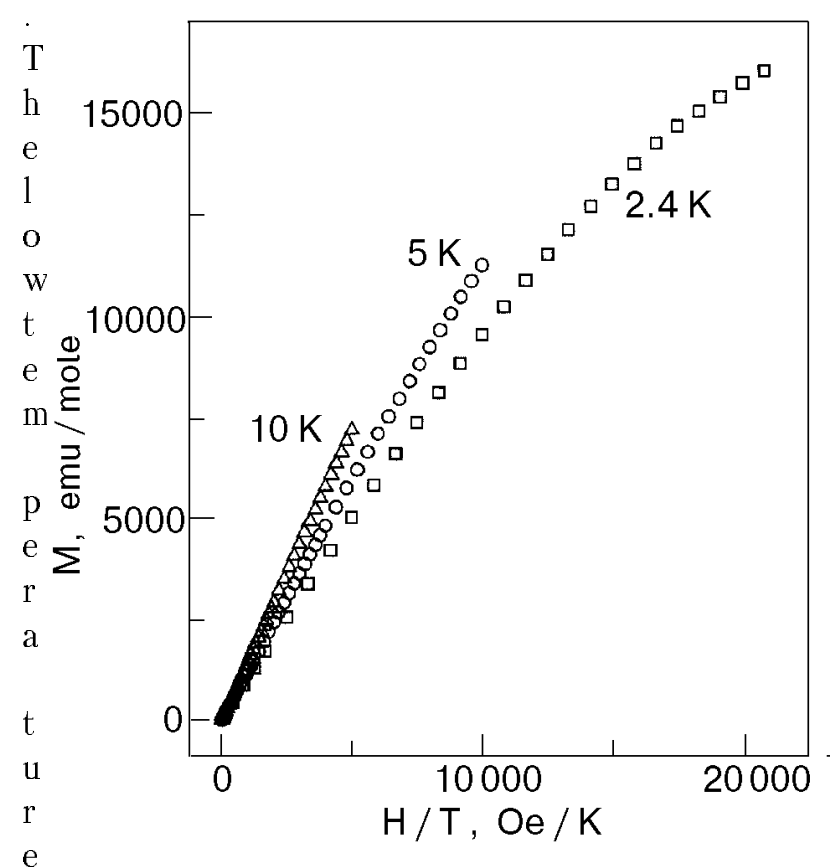

rfig. 3. Magnetization curves of $\mathrm{Ce}_{3} \mathrm{Pd}_{20} \mathrm{Ge}_{6}$ at different temperatures.

$\mathrm{g}$

netic susceptibility (at 12 and 2 Oe) is displayed in Fig. 2. Curves $\chi(T)$ clearly demonstrate a magnetic anomaly near $T_{1} \approx 2.8 \mathrm{~K}$. Below $T_{1}$ the increase in the susceptibility slows down noticeably which excludes a ferromagnetic type of this anomaly. Wherever, no an evidence of the magnetic hysteresis was observed in magnetization curves measured at $2 \mathrm{~K}$. Furthermore, as may be seen from Fig. 3, the effective magnetic moment (per $\mathrm{Ce}^{3+}$ ion) decreases with lowering of the temperature from 10 to $2.4 \mathrm{~K}$.

It should be noted that increase of the magnetic field at low temperatures suppresses the magnetic susceptibility [see Fig. 2, compare $M / H$ at 12 and 2 Oe (inset)]. This is a characteristic feature of spin-glass systems. In addition, a distinct remanent magnetic moment (RMM) was detected at low temperatures. The inset of Fig. 4 demonstrates the temperature dependence of RMM in $\mathrm{Ce}_{3} \mathrm{Pd}_{20} \mathrm{Ge}_{6}$ near $T_{1}$. To obtain these data the sample was cooled down to $2 \mathrm{~K}$ at the zero external magnetic field, then the magnetic field of 100 Oe was applied for a short time and next it was turned off. On subsequent heating, the RMM of the sample quickly fall down at temperature range from 2.4 to $3 \mathrm{~K}$ and fall down lower at temperatures above $3 \mathrm{~K}$. The existence of RMM also could indicate an «spinglass» (SG) nature of low temperature magnetism in $\mathrm{Ce}_{3} \mathrm{Pd}_{20} \mathrm{Ge}_{6}$. This point of view is supported by Fig. 4 which shows the difference $\Delta M$ between magnetic moments of $\mathrm{Ce}_{3} \mathrm{Pd}_{20} \mathrm{Ge}_{6}$ measured during zero field cooling (ZFC) and field cooling (FC) 


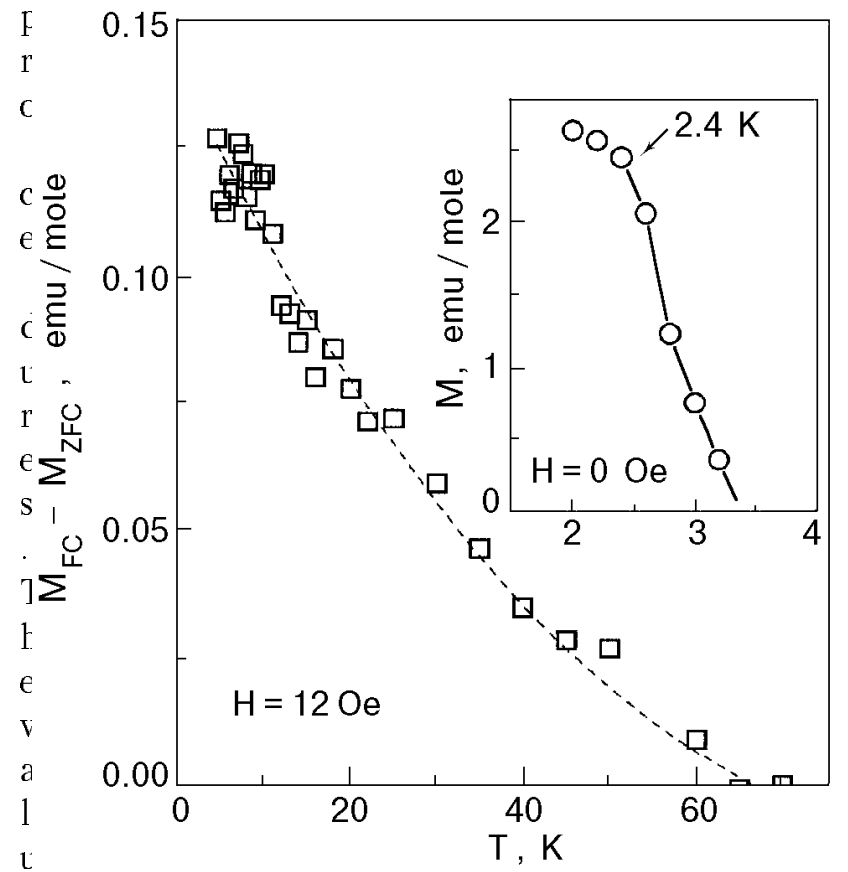

$\mathrm{e}^{F i g .}$ 4. The difference between the FC and ZFC magnetic moment of $\mathrm{Ce}_{3} \mathrm{Pd}_{20} \mathrm{Ge}_{6}$ versus the temperature. The inset shows $f$ the temperature dependence of the remanent magnetic moment below $4 \mathrm{~K}$.

c

learly tends to zero at temperatures above $T_{2} \approx 60 \mathrm{~K}$.

\section{Discussions}

Since 1995, when $\mathrm{Ce}_{3} \mathrm{Pd}_{20} \mathrm{X}_{6}$ was synthesized [5], magnetic properties of this compound rave been investigated in works [2-4,6-8]. It was found that the possibility of detecting magnetic anomalies depends strongly on the applied magnetic field. Thus, the cusp in the ac-magnetic susceptibility of $\mathrm{Ce}_{3} \mathrm{Pd}_{20} \mathrm{Si}_{6}$ at $0.15 \mathrm{~K}$ is suppressed by the weak static magnetic field [7]. The magnetic behavior of $\mathrm{Ce}_{3} \mathrm{Pd}_{20} \mathrm{Si}_{6}$ at high temperatures $(4-100 \mathrm{~K})$ is also sensitive to the static magnetic field [3]. This evident field-dependence on me applied magnetic field explains why the magnetic anomaly at $50 \mathrm{~K}$ in $\mathrm{Ce}_{3} \mathrm{Pd}_{20} \mathrm{Si}_{6}$ [3] and that at $2.7 \mathrm{~K}$ in $\mathrm{Ce}_{3} \mathrm{Pd}_{20} \mathrm{Ge}_{6}$, observed in this work, were undetected at the magnetic field 3 KOe used in works [4,8].

The marked field sensitivity of magnetic susceptibility in $\mathrm{Ce}_{3} \mathrm{Pd}_{20} \mathrm{Si}_{6}$ was analyzed within the framework of the «molecule magnetism» model [3]. This model could explain also a «spin-glass» magnetic behavior in $\mathrm{Ce}_{3} \mathrm{Pd}_{20} \mathrm{Si}_{6}$. According to [5] and the «molecule magnetism» model, there are two non-equivalent $\mathrm{Ce}$ positions ( $\mathrm{Ce} 1$ and $\mathrm{Ce} 2)$ in the crystal lattice of $\mathrm{Ce}_{3} \mathrm{Pd}_{20} \mathrm{X}_{6}$ [3]. First type (Ce1) ions form face-centred «large» cube and second type (Ce2) ones make up a «small» cube inside the
${ }_{\mathrm{r}}$ Fig. 5. The unit cell of $\mathrm{Ce}_{3} \mathrm{Pd}_{20} \mathrm{Ge}_{6}$ crystal structure. Only Ce positions are shown.

$\mathrm{r}$

omagnetic exchange interactions dominate over others, the could result in the formation of «superparamagnetic cubes» (SPC) containing eight $\mathrm{Ce} 2$ ions [3]. Anomaly at $50-60 \mathrm{~K}$ in $\mathrm{Ce} 2-\mathrm{Ce} 2$ can be attributed to antiferromagnetic (AF) ordering of SPC [3]. In this case Ce1 moments, placed between two SPC, undergo magnetic frustration. It is well known that magnetic frustration and spin-glass behavior are intimately related [9]. Non-freezing cerium magnetic moments are capable of bung effective Kondo's scattering centers [3]. Analogous frustration of exchange interactions may also takes place in $\mathrm{Ce}_{3} \mathrm{Pd}_{20} \mathrm{Ge}_{6}$. Within the framework of this model in the temperature range $50-60 \mathrm{~K}$ there may occur the magnetic transition of SPC to antiferromagnetic state in $\mathrm{Ce}_{3} \mathrm{Pd}_{20} \mathrm{Si}_{6}$ and to the «freezing» state (partial or complete) in $\mathrm{Ce}_{3} \mathrm{Pd}_{20} \mathrm{Ge}_{6}$. This characteristic temperature is determined mainly by Ce2-Ce2 exchange interactions which should be close in both compounds. From this point of view the second magnetic anomaly at $T_{1}$ in $\mathrm{Ce}_{3} \mathrm{Pd}_{20} \mathrm{Ge}_{6}$ is due to «freezing» of some Ce1 magnetic moments (see below).

Figure 5 shows the unit cell of $\mathrm{Ce}_{3} \mathrm{Pd}_{20} \mathrm{Ge}_{6}$ crystal structure (only Ce positions are presented). The analysis of an arrangement of cerium atoms relative to SPC reveals two different positions of $\mathrm{Ce} 1$ ions. These are $\mathrm{Ce}^{\prime}$ ' positions, which are located at corners of «large" cube, and Ce1" ones at centers of the faces. Because of their unequivalent location near SPC these Ce1 subsystems may undergo magnetic ordering at different tempera- 
tures. It is possible that $T_{1}$ is the temperature of «freezing» in one of these subsystem. This could result in the termination of Kondo-like increase of the electrical resistivity near $2 \mathrm{~K}$ [4]. Other Ce1 subsystem could have a transition to the antiferromagnetic phase under cooling below $1 \mathrm{~K}$ [4].

In conclusions, we suppose that $\mathrm{Ce}_{3} \mathrm{Pd}_{20} \mathrm{X}_{6}$ ( $\mathrm{X}=\mathrm{Ge}, \mathrm{Si}$ ) system could be considered as antiferromagnetic with a few $4 f$ magnetic subsystems and a strong frustration of exchange interactions. This could explain the anomalous magnetic behaviour (including «spin-glass» one) and coexistence of magnetic and Kondo-like properties at the same temperature region.

This work was partially supported by the Russian Fundamental Research Foundation (grant $95 / 02 / 04340$ and $95 / 03 / 09647)$.

1. Yu. Izyumov, M. Katsnelson, and Yu. Skryabin, Itinerant electron magnetism, Nauka, Moscow (1994).
2. Yu. P. Gajdukov, Yu. A. Koksharov, Yu. V. Kochetkov, J. Mirkovic, and V. N. Nikiforov, Pis'ma $v$ ZhETF 61, 385 (1995); [JETP Lett. 61, 381 (1995)].

3. V. N. Nikiforov, Yu. A. Koksharov, J. Mirkovic, and Yu. V. Kochetkov, JMMM 163, 184 (1996).

4. J. Kitagawa, N. Takeda, and M. Ishikawa, Phys. Rev. B53, 5101 (1996).

5. A. V. Gribanov, Yu. D. Seropegin, and O. I. Bodak, J. Alloys and Compounds 204, L9 (1994).

6. J. Kitagawa, N. Takeda, M. Ishikawa, T. Yushida, A. Ishiguro, and T. Komatsubara, in: Conf. Strongly Correlated Electron Systems, August 19-22, Zurich (1996) (in print).

7. N. Takeda, J. Kitagawa, and M. Ishikawa, in: Conf. on Strongly Correlated Electron Systems, August 19-22, Zurich (1996) (in print).

8. N. Takeda, J. Kitagawa, and M. Ishikawa, J. Phys. Soc. Jpn. 64, 387 (1995).

9. S. Ozeroff and P. Keezom, in: Semiconductors and Semimetals, Diluted Magnetic Semiconductors Volume, v. 25, Jacek K. Furdyna and Jacek Kossut (eds.), Academic Press Inc. (1988). 